\title{
A Review Study for Electrocardiogram Signal Classification
}

\author{
Lana Abdulrazaq Abdullah", ${ }^{1,2}$, Muzhit Shaban Al-Ani ${ }^{3}$ \\ ${ }^{1}$ Department of Computer Science, College of Science and Technology, University of Human Development, Sulaymaniyah, \\ KRG, Iraq, ${ }^{2}$ Department of Computer, College of Science, University of Sulaimani, Sulaymaniyah, KRG, Iraq, ${ }^{3}$ Department of \\ Information Technology, College of Science and Technology, University of Human Development, Sulaymaniyah, KRG, Iraq
}

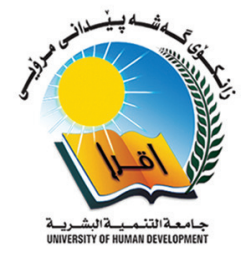

\section{A B S T R A C T}

An electrocardiogram (ECG) signal is a recording of the electrical activity generated by the heart. The analysis of the ECG signal has been interested in more than a decade to build a model to make automatic ECG classification. The main goal of this work is to study and review an overview of utilizing the classification methods that have been recently used such as Artificial Neural Network, Convolution Neural Network (CNN), discrete wavelet transform, Support Vector Machine (SVM), and K-Nearest Neighbor. Efficient comparisons are shown in the result in terms of classification methods, features extraction technique, dataset, contribution, and some other aspects. The result also shows that the CNN has been most widely used for ECG classification as it can obtain a higher success rate than the rest of the classification approaches.

Index Terms: Artificial neural network, Convolution neural network, Discrete wavelet transform, Support vector machine, K-nearest neighbor

\section{INTRODUCTION}

An electrocardiogram (ECG) is simply a recording of the electrical activity generated by the heart [1]. The heart produces the electrical activity that measures by a medical test called an ECG, which identifies the cardiac abnormality [2]. A heart produces tiny electrical impulses that spread through the heart muscle [3]. An ECG all data about the electrical activity of the heart records and shows on a paper by an ECG machine [4]. Then, a medical practitioner interprets this data; ECG leads to find the cause of symptoms of chest pain and also leads to detect abnormal heart rhythm [5].

\begin{tabular}{|l|l|}
\hline \multicolumn{2}{|c|}{ Access this article online } \\
\hline DOI:10.21928/uhdjst.v4n1y2020.pp103-117 & $\begin{array}{l}\text { E-ISSN: 2521-4217 } \\
\text { P-ISSN: 2521-4209 }\end{array}$ \\
\hline $\begin{array}{l}\text { Copyright @ 2020 Abdullah and Al-Ani. This is an open access article } \\
\text { distributed under the Creative Commons Attribution Non-Commercial } \\
\text { No Derivatives License 4.0 (CC BY-NC-ND 4.0) }\end{array}$ \\
\hline
\end{tabular}

An ECG signal has a total of five primary turns, counting $\mathrm{P}, \mathrm{Q}, \mathrm{R}, \mathrm{S}$, and $\mathrm{T}$ waves, plus the depolarization of the atria causes a small turn before atria contraction as the activation (depolarization) wave-front propagates from the Sino atria node through the atria [6]. The Q wave is a downward deflection after the $\mathrm{P}$ wave [7]. The $\mathrm{R}$ wave follows as an upward deflection, and the $\mathrm{S}$ wave is a downward deflection following the $\mathrm{R}$ wave [8]. Q, R, and $\mathrm{S}$ waves together indicate a single event [9]. Hence, they are usually considered to be QRS complex, as shown in Fig. 1 [10], [11].

The features based on the QRS complex are among the most powerful features for ECG analysis [13]. The QRS-complex is caused by currents that are generated when the ventricles depolarize before their contraction [14]. Although atrial depolarization occurs before ventricular depolarization, the latter waveform (i.e., the QRS-complex) has much higher amplitude, and atria depolarization is, therefore, not seen on an ECG. The T wave, which follows the $\mathrm{S}$ wave, is ventricular depolarization, where the heart muscle prepares for the next

Corresponding author's e-mail: Lana Abdulrazaq Abdullah, Department of Computer Science, College of Science and Technology, University of Human Development, Sulaymaniyah, KRG, Iraq, Department of Computer, College of Science, University of Sulaimani, Sulaymaniyah, KRG, Iraq. E-mail: lana.abdulla@uhd.edu.iq

Received: 05-02-2020 Accepted: 12-06-2020 Published: 29-06-2020 
ECG cycle [15]. Finally, the $U$ wave is a small deflection that immediately follows the $\mathrm{T}$ wave. The $\mathrm{U}$ wave is usually in the same direction as the $T$ wave [16].

There are different kinds of arrhythmias, and each kind is associated with a pattern, and as such, it is possible to recognize and classify it [17]. The arrhythmias can be categorized into two major classes; the first class consists of arrhythmias formed by a single irregular ECG signal, herein called morphological arrhythmia, the other type consists of arrhythmias formed by a set of irregular heartbeats, herein called rhythmic arrhythmias [18].

The main problem in the process of identifying and classifying arrhythmias ECGs is that an ECG signal can vary for each person, and sometimes different patients have separate ECG morphologies for the same disease [19]. Moreover, two various diseases could have approximately the same properties on an ECG signal [20]. These problems cause some difficulties in the issue of heart disease diagnosis [21].

Furthermore, the ECG records analysis is complicated for a human due to fatigue; an alternative way for automatic classification is computerization techniques [22]. For arrhythmia classification from the signal received by ECG device needed an automated system that can be divided into three main steps, as follows first: Pre-processing, next: Feature extraction and finally: Classification, as shown in Fig. 2 [23].

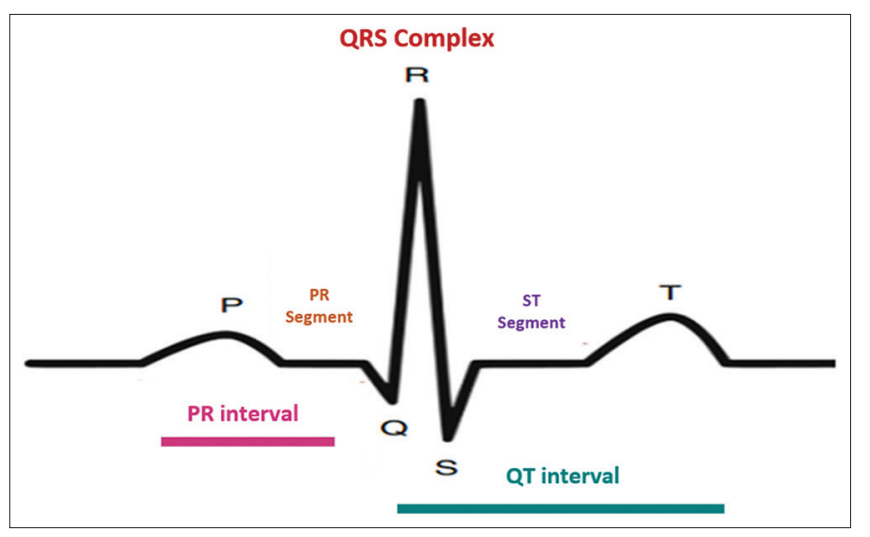

Fig. 1. A typical electrocardiogram signal [12].
ECG signals may contain several kinds of noises, which can affect the extraction of features used for classification; therefore, the pre-processing step is necessary for removing the noises [24]. Researchers have applied different preprocessing techniques for ECG classification. For noise removal, techniques such as low pass linear phase filter and linear phase high pass filters, etc., are used [25]. Some methods, such as median filter, linear phase high pass filter, and mean median filter are used baseline adjustment [26].

After the pre-processing step, extracting different ECG features then used as inputs to the classification model [27]. Feature extraction techniques used by researchers are discrete wavelet transform (DWT), continuous wavelet transform, discrete cosine transform (DCT), discrete Fourier transform, principal component analysis (PCA), Pan-Tompkins algorithm, and independent component analysis (ICA) [28].

When the set of features has been defined from the heartbeats, models can be built from these data using artificial intelligence algorithms from machine learning and data mining domains for arrhythmia heartbeat classification. The most popular techniques employed for this task and found in the literature are artificial neural networks (ANN), convolution neural network (CNN), DW'T, support vector machines (SVM), decision tree (DT), Bayesian, Fuzzy, linear discriminate analysis (LDA), and k-nearest neighbors (KNN) [29].

Many surveys on ECG analysis and classification have been published. In Karpagachelvi [30] surveyed the most effective features for ECG analysis and classification as ECG. Features play a significant role in diagnosing most of the cardiac diseases. Nasehi and Pourghassem [31] provided a survey of variance types of seizure detection algorithms and their potential role in diagnostic. Various machinelearning approaches for ECG analysis and classification were reviewed in Roopa and Harish [23]. A comprehensive review was published in 2018, which includes a literature on ECG analysis mostly from the past decade, and most of the major aspects of ECG analysis were addressed such as preprocessing, denoising, feature extraction, and classification methods [16] (Previous works on ECG survey paper, Reviewer 2).

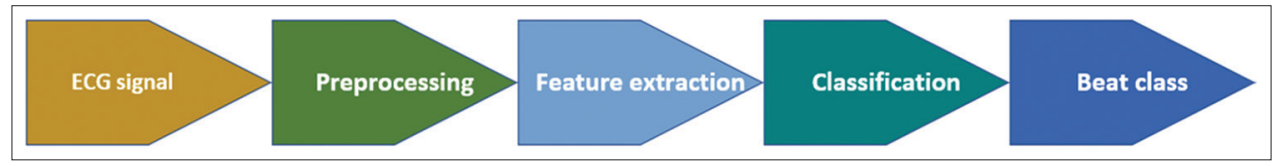

Fig. 2. General diagram of electrocardiogram classification. 
The main purpose of this work is to review most of the common techniques that have been used mostly from the past 5 years. Moreover, the paper can be useful for the other researchers in identifying any issue in ECG classification and analyzing the research area as many aspects of the methods are addressed. (This section is the main purpose of the paper (reviewer 3)).

The section of this paper is ordered as follows: Section 2 contains Classification Techniques, and then Section 3 provides of Discussion, and finally, Section 4 presents the Conclusion.

\section{CLASSIFICATION}

A lot of pathological information about a patient's heart processes can be obtained by studying the ECG signal [32]. There are many approaches have been developed to classify heartbeats as it is essential for the detection of an arrhythmia [33]. Arrhythmias can be divided into two parts, which are life-threatening and non-life-threatening arrhythmias, a long-term ECG classification is required for the diagnosis of non-life-threatening arrhythmias that could be time-consuming and impractical, automatic algorithms exhibit a great aid. Consequently, automatic ECG classification of arrhythmias is one of the most worth studying in the world [34].

There are various classifiers that have been used for ECG classification task. In this paper, most common ECG classification methods are reviewed that were proposed since 2016-2020, these classification methods can be mainly clustered based on the classifiers into several categories such as ANNs, CNN, kNN, SVM, and DWT. All of the reviewed papers were accessed by three well-known publishers, which are IEEE, ScienceDirect, and Springer. (This section was wrote about why and how the authors select the papers for this state (Reviewer 2 and reviewer 3).

Different types of classification techniques are studied to classify ECG data under the variance features, as there are plenty of features in the ECG signal that can be extracted. Some of the classification methods are addressed below.

\subsection{ANN}

The ANN is an adaptive system with exciting features such as the ability to adapt, learn, and summarize; because ANN's parallel processing, self-organizing, fault-tolerant, and adaptive capabilities make it capable of solving many complex problems, ANN is also very accurate in the classification and prediction of outputs [35]. The neural network $(\mathrm{NN})$ consists of the number of layers; the initial layer has an association as of the system input, and the end layer gives the output of the network [36]. NN s having hidden layers and sufficient neurons can be applied to any limited input-output mapping trouble [37]. The NN model consists of an input layer, the hidden layer, and output layer, as shown in Fig. 3 [38].

Many kinds of literature are published related to the ECG classification based on ANN. Below some of these new approach:

Chen et al. (2016) proposed a wavelet-based ANN (W-ANN) method that was based on the wavelet transform. The result illustrated that the W-ANN can provide lower computing time such that reduction time was $49 \%$ and cleaner ECG input signal. The method was implemented on the data MIT-BIH arrhythmia database and real ECG signal measurement [39].

Boussaa et al. (2016) presented the design of a cardiac pathologies detection system with high precision of calculation and decision, which consists of the mel-frequency coefficient cepstrum algorithms such as fingerprint extractor (or features) of the cardiac signal and the algorithms of ANN multilayer perceptron (MLP) type MLP classifier as fingerprints extracted into two classes: Normal or abnormal. The design and testing of the proposed system are performed on two types of data extracted from the MITBIH database: A learning base containing labeled data (ECG normal and abnormal) and another test base containing no-labeled data. The experimental results were shown that the proposed system combines the respective advantages of the descriptor mel-frequency cepstrum coefficient and the MLP classifier [40].

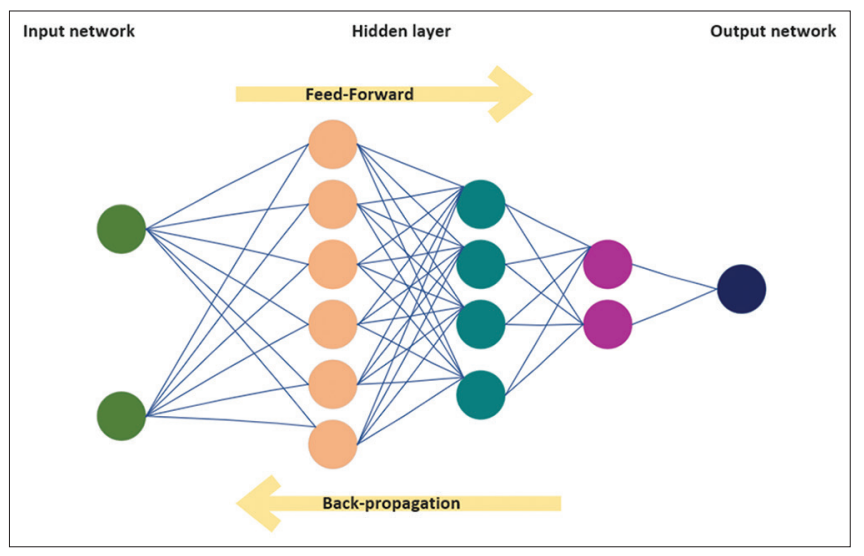

Fig. 3. Artificial neural network. 
Savalia et al. (2017) distinguished between normal and abnormal ECG data using signal processing and NNs toolboxes in Matlab. Data, which were downloaded from an ECG database, PhysioBank, were used for learning the NN. The feature extraction method was also used to identify variance heart diseases such as bradycardia, tachycardia, firstdegree atrioventricular (AV), and second-degree AV. Since ECG signals were very noisy, signal processing techniques were applied to remove the noise contamination. The heart rate of each signal was calculated by finding the distance between R-R intervals of the signal. The QRS complex was used to detect AV blocks. The result showed that the algorithm strongly distinguished between normal and abnormal data as well as identifying the type of disease [41].

Wess et al. (2017) presented field-programmable gate array (FPGA)-based ECG arrhythmia detection using an ANN. The objective was to implement a NN-based machinelearning algorithm on FPGA to detect anomalies in ECG signals, with better performance and accuracy (ACC), compared to statistical methods. An implementation with PCA for feature reduction and a MLP for classification, proved superior to other algorithms. For implementation on FPGA, the effects of several parameters and simplification on performance, ACC, and power consumption were studied. Piecewise linear approximation for activation functions and fixed-point implementation was effective methods to reduce the number of needed resources. The resulting NN with 12 inputs and six neurons in the hidden layer, achieved, in spite of the simplifications, and the same overall ACC as simulations with floating-point number representation. An ACC of $99.82 \%$ was achieved on average for the MIT-BIH database [42].

Pandey et al. (2018) compared three different ANN models for classification normal and abnormal signals and using University of California, Irvine ECG 12 lead signal data. This work had used methods, namely, back propagation (BP) network, radial basis function (RBF) networks, and recurrent neural network (RNN). RNN models have shown better analysis results. ACC for testing classification was $83.1 \%$. This result was better than some work, using the same database [43].

Sannino and Pietro (2018) proposed an approach based on a deep neural network (DNN) for the automatic classification of abnormal ECG beats, differentiated from normal ones. DNN was developed using the Tensor Flow framework, and it was composed of only seven hidden layers, with 5, 10, 30, $50,30,10$, and 5 neurons, respectively. Comparisons were made among the proposed model with 11 other well-known classifiers. The numerical results showed the effectiveness of the approach, especially in terms of ACC [44].

Debnath et al. (2019) proposed two schemes; at first, the QRS components have been extracted from the noisy ECG signal by rejecting the background noise. This was done using the Pan-Tompkins algorithm. The second task involved the calculation of heart rate and detection of tachycardia, bradycardia, asystole, and second-degree AV block from detected QRS peaks using MATLAB. The results showed that from detected QRS peaks, and arrhythmias, which are based on an increase or decrease in the number of QRS peaks, the absence of a QRS peak, could be diagnosed. The final task is to classify the heart abnormalities according to previously extracted features. The BP trained feed-forward NN has been selected for this research. Here, data used for the analysis of ECG signals are from the MIT database [45].

Abdalla et al. (2019) presented that approach was developed based on the non-linearity and nonstationary decomposition methods due to the nature of the ECG signal. Complete ensemble empirical mode decomposition with adaptive noise (CEEMDAN) was used to obtain intrinsic mode functions (IMFs). Established on those IMFs, four parameters have been computed to construct the feature vector. Average power, coefficient of dispersion, sample entropy, and singular values were calculated as parameters from the first six IMFs. Then, ANN was adopted to apply the feature vector using them and classify five different arrhythmia heartbeats downloaded from PhysioNet in the MIT-BIH database. The performance of the CEEMDAN and ANN was better than all existing methods, where the sensitivity (SEN) is $99.7 \%$, specificity (SPE) is $99.9 \%$, ACC is $99.9 \%$, and receiver operating characteristic (ROC) is $01.0 \%$ [46].

\subsection{Convolutional Neural Network (CNN)}

The CNN is the most common technique to classify ECG, $\mathrm{CNN}$ is mainly composed of two parts, feature extraction and classification [47]. The section of feature extraction is responsible for extracting effective features from the ECG signals automatically, while the part of classification is in charge of classifying signals accurately by making use of the extracted features, as shown in Fig. 4 [48].

Many approaches are published the ECG classification based on $\mathrm{CNN}$. Below some of these update works:

Zubair et al. (2016) proposed a model which was integrated into two main parts, feature extraction, and classification. 


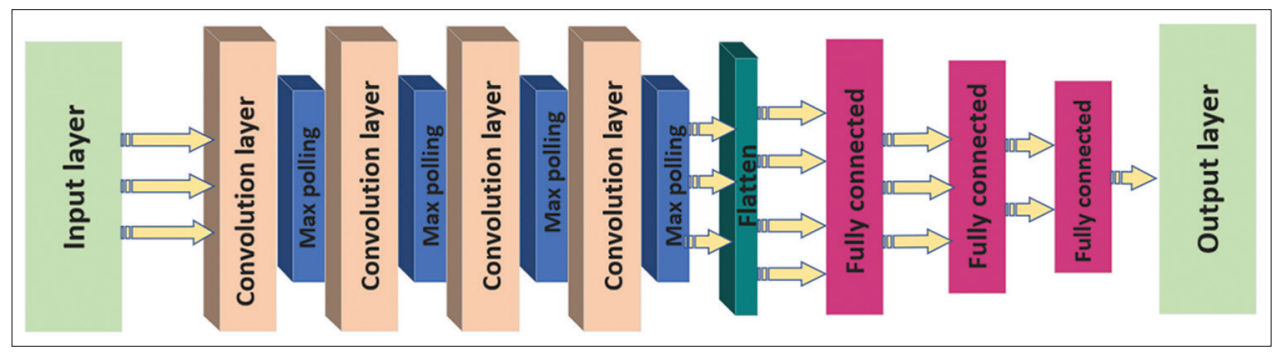

Fig. 4. Typical convolution neural network structure.

The model automatically remembers a suitable feature representation from raw ECG data and thus negates the need for hand-crafted features. Using small and patientspecific training data, the proposed classification system efficiently classified ECG beats into five different classes. ECG signal from 44 recordings of the MIT-BIH database is used to assess the classification performance, and the results demonstrate that the proposed approach achieves a significant classification ACC and superior computational efficiency than most of the state-of-the-art methods for ECG signal classification [49].

Yin et al. (2016) proposed a system that applies the impulse radio ultra-wideband radar data as additional information to assist the arrhythmia classification of ECG recordings in the slight motion state. Besides, this proposed system employs a cascaded CNN to achieve an integrated analysis of ECG recordings and radar data. The experiments are implemented in the Caffe platform, and the result reaches an ACC of $88.89 \%$ in the slight motion state. It turns out that this proposed system keeps a stable ACC of classification for normal and abnormal heartbeats in the slight motion state [50].

Oh et al. (2017) designed a nine-layer deep CNN DCNN to identify five different categories of heartbeats in ECG signals automatically. The test was applied in original and ECG signals that were derived from the available database. The set was artificially augmented for removing high-frequency noise. The $\mathrm{CNN}$ model was trained to utilize the augmented data and obtained an ACC of $93.47 \%$ and $94.03 \%$ in the identification of heartbeats in noise-free and original ECGs [51].

Zhai and Tin (2018) proposed an approach based on the CNN model with a different structure. The model was improved SEN, and positive predictive rate for $\mathrm{S}$ beats by more than $12.2 \%$ and $11.9 \%$, respectively. The system provided a fully automatic tool and reliable to detect the arrhythmia heartbeat without any manual feature extraction or any expert assistant [52].
Zhang et al. (2019) introduced a new pattern recognition method in ECG data using DCNN. Different from past methods that utilized learn features or hand-crafted features from the raw signal domain, the proposed method was learned the features and classifiers from the time-frequency domain. First, the ECG wave signal was transformed into the time-frequency domain using the Short-Time Fourier Transform. Then, several scale-specific DCNN models were trained on ECG samples of a specific length. Eventually, an online decision fusion method was proposed to fuse decisions at different scales into a more accurate and stable one [53].

Wang (2020) proposed a novel approach for the automated atria fibrillation (AF) detection based DNN, which was built 11-layers. The network structure was combined using a modified Elman neural network (MENN) and CNN. Ten-Fold cross-validation was conducted to evaluate the classification performance of the model on the MIT-BIH AF database. The result confirmed that the model yielded excellent classification performance with the ACC, SEN, and SPE of $97.4 \%, 97.9 \%$, and $97.1 \%$, respectively [54].

Yao et al. (2020) designed model attention based on timeincremental CNN (ATI-CNN); a DNN model could obtain both spatial and temporal fusion of information from ECG signals using integrating $\mathrm{CNN}$. The features were flexible input length, halved parameter amount as well as more than $90 \%$ computation reduction in real-time processing. The experiment result showed that ATI-CNN achieved an overall classification rate of $81.2 \%$ compared to VGGNET that is a classical 16-layer CNN, ATI-CNN achieved ACC increases of $7.7 \%$ in average, and up to $26.8 \%$ in detecting paroxysmal arrhythmias [55].

\subsection{DWT}

The DWT is used to recognize and diagnose the ECG signals and widely used in signal processing [56]. A perfect time resolution is the main advantage of DWT [57]. It provides good frequency resolution at low frequency and good resolution at high frequency [58]. The DW'T can reveal the 
local characteristics of the input signal because of this great time and frequency localization ability [59].

Many kinds of literature are published related to the ECG classification based on DW'T. Below some of these new approach:

Desai et al. (2015) described a machine learning-based approach for detecting five classes of ECG arrhythmia beats based on DWT features. Moreover, ICA was used to comprise dimensionality reduction. ANOVA approach was used to select significant features, and ten-fold crossvalidation was used to perform SVM. The experiment was conducted on MIT-BIH arrhythmia, which is grouped into five classes of arrhythmia beats, namely, non-ectopic $(\mathrm{N})$, ventricular ectopic $(\mathrm{V})$, supraventricular ectopic $(\mathrm{S})$, fusion $(\mathrm{F})$, and unknown (U). Using SVM quadratic kernel classified ECG features with an overall average ACC of $98.49 \%$ [60].

Saraswat (2016) explored diverse possibilities of the decomposition using the DWT method to classify Wolff Parkinson White Syndrome ECG signals. In this work, ECG signals are discretely sampled till the $5^{\text {th }}$ resolution level of the decomposition tree using DWT with Daubechies wavelet of order 4 (db4), which helps in smoothing the feature more appropriate for detecting changes in signals. The MIT-BIH database was used for some experimental results [61].

Alickovic and Subasi (2016) noted that RF classifiers achieved superior performances compared to DT methods using ten-fold cross-validation for the ECG datasets. The results suggested that further significant developments in words of classification ACC could be accomplished by the proposed classification system. Accurate ECG signal classification was the major requirement for the detection of all arrhythmia types. Performances of the proposed system were evaluated on two different databases, namely, MIT-BIH database and St. Petersburg Institute of Cardiological Techniques 12-lead Arrhythmia Database. For the MIT-BIH database, the RF classifier generated an overall ACC of $99.33 \%$ against 98.44 and $98.67 \%$, respectively. For St. Petersburg Institute of Cardiological Technics 12-lead Arrhythmia Database, RF classifier yielded a general ACC for the C4.5 and CART classifiers of $99.95 \%$ against $99.80 \%$ for both C4.5 and CART classifiers, respectively. The merged model with multiscale PCA de-noising, DW'T, and RF classifier also achieves good performance for MIT-BIH database with the area under the ROC curve (area under the curve [AUC]) and F-measure equal to 0.999 and 0.993 and 1 and 0.999 for and St. Petersburg Institute of Cardiological Technics 12-lead Arrhythmia Database, respectively. The results demonstrated that the proposed system was able for reliable classification of ECG signals and to help the clinicians to make an accurate diagnosis of cardiovascular disorders (CVDs) [62].

Pan et al. (2017) proposed a comprehensive approach based on random forest techniques and discrete wavelet for arrhythmia diagnosis. Specifically, DWT was used to remove high-frequency noise and baseline drift, while DWT, autocorrelation, PCA, variances, and other mathematical methods are used to extract frequency-domain features, time-domain features, and morphology features. Moreover, an arrhythmia classification system was developed, and its availability was verified that the proposed scheme could significantly be used for guidance and reference in clinical arrhythmia automatic classification [63].

Sahoo (2017) proposed an improved algorithm to find QRS complex features based on the wavelet transform to classify four kids of ECG beats: Normal $(\mathrm{N})$, left bundle branch block (LBBB), right bundle branch block (RBBB), and Paced beats $(\mathrm{P})$; using NN and SVM classifier. Model performance was evaluated in terms of SEN, SPE, and ACC for 48-recorded ECG signals obtained from the MIT-BIH arrhythmia database. The proposed procedure achieved high detection efficiency with a low error rate of $0.42 \%$ when detecting the QRS compound. The classifier fixed its superiority with an average ACC of $96.67 \%$ and $98.39 \%$ in SVM and NN, respectively. The classification ACC of the SVM approach proves superior for the proposed method to that of the NN classifier with extracted parameters in detecting ECG arrhythmia beats [64].

Ceylan (2018) studied a model based on spared coefficients of the signals that were achieved by employing sparse representation algorithms and dictionary learning. The obtained coefficients were utilized in the weight update process of three different classification approaches, which were created using SVM, AdaBoost, and LDA algorithms. In the first step, the proposed Dictionary Learning (DL) based AdaBoost classifiers isolated the ECG signals. Then, the selected feature was applied to ECG signals, and six different feature subsets were obtained by DWT, T-test, Bhattacharyya, First Order Statistics (FOS), Wilcoxon test, and Entropy methods. The subscription of objects was used as a new dataset. The classification process is performed according to the proposed method, and satisfactory results are obtained. The best classification ACC was received at $99.75 \%$ using the proposed commercial-based terminology method called DL-AdaBoost-SVM for the subset of attributes obtained using the DWT and Wilcoxon test methods [65]. 
Tea and Vladan (2018) proposed a novel framework that combined the theory of compressive sensing and random forests to achieve reliable automatic cardiac arrhythmia detection. Moreover, it evaluated the characterization power of DCT, DWT, and FFT data transformations to extract significant features that can bring an additional boost to the classification performance. The experiments conducted on the MIT-BIH benchmark arrhythmia database, the result demonstrated that DWT based features exhibit better returns compared to the feature extraction technique for a relatively small number of random projected coefficients. Furthermore, due to its low-complexity, the proposed model could be implemented for practical applications of real-time ECG monitoring [66].

Zhang et al. (2019) proposed a lightweight approach to classify five types of cardiac arrhythmia; namely, normal beat $(\mathrm{N})$, premature ventricular contraction $(\mathrm{PVC})(\mathrm{V})$, atria premature contraction (APC) (A), RBBB beat (R), and LBBB beat $(\mathrm{L})$. The mixed method of frequency analysis and Shannon entropy was applied to extract appropriate statistical features. The information gain criterion was manipulated for selecting features. The selected features were then fed to the input of Random Forest, KNN, and J48 for classification. To evaluate classification performance, tenfold cross-validation was used to verify the effectiveness of our method. Experimental results showed that the Random Forest classifier demonstrates significant performance with the SPE of $99.5 \%$, the highest SEN of $98.1 \%$, and the ACC of $98.08 \%$, outperforming other representative approaches for automated cardiac arrhythmia classification [67].

Kora et al. (2019) showed that an algorithm to detect atrial fibrillation (AF) in the ECG signal is developed. For correct detection of AF, pre-processing and feature extraction of the ECG signal shall be performed before it detects AF. After considering the ECG signal from the database, in the preprocessing stage, denoising of the ECG signal is carried out to obtain a clean ECG signal. After pre-processing, before feature extraction, $R$ peak detection is carried out for the signal. Since $\mathrm{R}$ peak has the highest amplitude, and therefore, it is detected in the first round, and subsequently location of other peaks of the ECG signals is performed. After completing, pre-processing and feature extraction using DWT applied based on inverted $\mathrm{T}$ wave logic and ST-segment elevation. Our classification algorithm was demonstrated to successfully acquire, analyze, and interpret ECGs for the presence of AF, indicating its potential to support $\mathrm{m}$-Health diagnosis, monitoring, and management of therapy in AF patients [68].

\subsection{SVM}

SVM is a learning algorithm that has many good properties. It is associated with data analysis and recognizes the pattern. SVM uses a linear discriminate function for classification; however, non-linear classification can also be done if a nonlinear kernel is used [69]. SVM performs well in real-time situations, robust, easy to understand. While compared to other classifiers [30]. A classification task typically requires the knowledge about the data to be classified; hence, the classifier must be trained before classifying any data [70]. One of the main advantages of the SVM classifier is that it automatically finds the support vectors for better classification [71]. Majorly, in every case the performance of SVM depends on the affected kernel function selection [72].

Many types of research are published in the ECG classification based on the SVM. Below some of these recent studies:

Elhaj et al. (2016) investigated a combination of linear and non-linear features to improve the classification of ECG data. In the study, five types of beat classes of arrhythmia as recommended by the Association for Advancement of Medical Instrumentation are analyzed: Non-ectopic beats $(\mathrm{N})$, supra-ventricular ectopic beats (S), ventricular ectopic beats $(\mathrm{V})$, fusion beats $(\mathrm{F})$, and unclassifiable and paced beats $(\mathrm{U})$. The characterization ability of non-linear features such as high order statistics and cumulants and non-linear feature reduction methods such as ICA is combined with linear features, namely, the PCA of DWT coefficients. The features are tested for their ability to differentiate different classes of data using different classifiers, namely, the SVM and NN methods, with tenfold cross-validation. This method can classify the N, S, V, F, and U arrhythmia classes with high ACC (98.91\%) using a combined SVM and RBF method [73].

Arjunan (2016) reported that statistics features could be useful for categorizing the ECG signals. Like the first, the signal has been passed from the de-noising process as a pre-processing. Then, the following statistics features such that mean, variance, standard deviation, and skewness are extracted from the signal. SVM was implemented to classify the ECG signal into two categories; normal or abnormal. The results show that the system classifies the given ECG signal with $90 \%$ SEN and SPE [74].

Smíšek et al. (2017) proposed method for automatic ECG classification to four classes (normal rhythm $[\mathrm{N}], \mathrm{AF}[\mathrm{A}]$, another rhythm $[\mathrm{O}]$, and noisy records $[\mathrm{P}])$. The SVM approach was involved in the two different stages in the model. In the first stage, SVM was used to extract the global 
features from the entire ECG signal. In the second stage, the features from the previous step were used to train the second SVM classifier. The cross-validation technique was used to evaluate both classifiers. The result showed that in Phase II of challenge, the total F1 score of the method was 0.81 and 0.84 within the hidden challenge dataset and training set, respectively [75].

Wu et al. (2017) developed a system for identifying excessive alcohol consumption. Three sensors were used to acquire signals regarding (ECG), intoxilyzers, and photoplethysmograph (PPG). Intoxilyzers were used to know alcohol consumption levels of participants before and after drinking. The signals were pre-processed, segmented, and subjected to feature extraction using specific algorithms to produce ECG and PPG training and test data. Using the ECG, PPG, and alcohol consumption data, the developed model was fast and accurate for the identification scheme using the SVM algorithm. Using the training data for training and the test data were applied to comfort the recognition performance of the trained SVMs. The identification performance of the proposed classifiers achieved $95 \%$ on average. In the approach, different feature combinations were tested to select the optimum technological configuration. Because the PPG and ECG features produce identical classification performance and the PPG features were more convenient to acquire, the technical setting based on PPG is preferable for developing smart and wearable devices for the identification of driving under the influence [76].

Venkatesan et al. (2018), ECG signal pre-processing and SVM -based arrhythmic beat classification is performed to categorize into normal and abnormal subjects. In ECG signal pre-processing, a delayed error normalized LMS adaptive filter is used to achieve high speed and low latency design with less computational elements. Since the signal processing technique is developed for distant healthcare systems, white noise removal is mainly focused. DWT is applied to the preprocessed signal for HRV feature extraction, and machinelearning techniques are used for performing arrhythmic beat classification. In this paper, the SVM classifier and other popular classifiers have been used on noise removed feature extracted signal for beat classification. The results show that the SVM classifier performs better than additional machine learning-based classifiers [77].

Liu et al. (2019) proposed an ECG arrhythmia classification algorithm based on CNN. They compared the CNN models with combining linear discriminant analysis (LDA) and SVM. All cardiac arrhythmia beats are derived from the MIT-BIH
Arrhythmia Database, which was classed into five groups according to the standard developed by the Association for the Advancement of Medical Instrumentation (AAMI). The training set and the testing set come from different people, and the correction of classification is $>90 \%$ [78].

\subsection{KNN}

The KNN algorithm is a simple machine-learning algorithm compared to similar machine learning approaches [79]. Most of the machine-learning algorithms work on the KNN algorithm [80]. KNN classifier is an instance-based learning method, which stores all training sample vectors [81]. It is a very simple and effective method, especially for highdimensional problems [82]. It classifies the new unknown test samples based on similar training samples [83]. The similarity measure is usually the Euclidean distance [84]. K-NN classifier was based on grouping of closest training points of data in the considered feature space. The majority of voters do the cluster to the nearest neighbor points [85].

Many approaches are published the ECG classification based on KNN. Below some of these new works:

Faziludeen and Sankaran (2016) presented a method for automatic ECG classification into two classes: Normal and PVC. The Evidential K-Nearest Neighbors (EKNN) was based on the Dempster Shafer Theory for classifying the ECG beats. RR interval features were used. The analysis was performed on the MIT-BIH database. The performance of EKNN was compared with the traditional KNN (maximum voting) approach. The effect of training data size was assessed using training sets of varying sizes. The EKNN based classification system was shown to out perform the KNN based classification system consistently [86].

Bouaziz et al. (2018) implemented an automatic ECG heartbeats classifier based on KNN. The segmentation of ECG signals has been performed by DWT. The considered categories of beats are normal (N), PVC, APC, RBBB, and LBBB. The validation of the presented KNN based classifier has been achieved using ECG data from MITBIH arrhythmia database. They have obtained the excellent classification performances, in terms of the calculated values of the SPE and the SEN of the classifier for several pathological heartbeats and the global classification rate, which is equal to $98,71 \%$ [87].

Khatibi and Rabinezhadsadatmahaleh (2019), a novel feature engineering method, was proposed based on deep learning and K-NNs. The features extracted were classified with 
different classifiers such as DTs, SVMs with different kernels, and random forests. This method has good performance for beat classification and achieves the average ACC of $99.77 \%$, AUC of $99.99 \%$, precision of $99.75 \%$, and recall of $99.30 \%$ using fivefold Cross-Validation strategy. The main advantage of the proposed method was its low computational time compared to training deep learning models from scratch and its high ACC compared to the traditional machine learning models. The strength and suitability of the proposed method for feature extraction are shown by the high balance between SEN and SPE [88].

\section{DISCUSSION}

The ECG classification, which shows the status of the heart and the cardiovascular condition, is essential to improve the patient's living quality. The main purpose of this work is to review the main techniques of ECG signal classification. In general, any structure of ECG classification can be divided into four stages. The first one is a preprocessing step, which is a crucial step in the ECG signal classification. For that reason, most well-known techniques are reviewed in this paper. The idea of using the preprocessing step and the combination of preprocessing techniques is to improve the performances of the model. The second step is extracting the most relevant information from the ECG signal, which represents the heart status. The step is called a feature extraction step. There is a vital challenge to extract efficient information that can be discriminated based on the variance status of the ECG signal. The success rate of the model can evaluate whether the feature contains valuable knowledge of the signal or not. The third step is named as the feature selection step. Time execution of the model is a crucial part and can be reduced using optimal features among the feature spaces. Many techniques have been adopted for reducing the dimensionality of the features. Some of the methods have been inspired by nature and the others, working based on the mathematical rules. The primarily focused step is selecting a machine-learning algorithm to classify the ECG features. Plenty of approaches has been used for this purpose. Most of the classifier methods are fed by the features, but $\mathrm{CNN}$ is supplied using the raw signal as $\mathrm{CNN}$ is a feature-less technique. ANN, CNN, DWT, KNN, and SVM are reviewed. All reviewed articles are downloaded from three trusted sources, IEEE, ScienceDirect, and Springer for 2015-2020. Tables 1-5 show the summarization of all the reviewed articles in term of what kind of machine-learning were used, how the methods were effective to the ECG

TABLE 1: Heartbeat methods classification based on ANN

\begin{tabular}{|c|c|c|c|c|c|}
\hline \multicolumn{6}{|c|}{ Artificial neural networks } \\
\hline Author (year) & Dataset & Purpose & Methods & Result & Remarks \\
\hline $\begin{array}{l}\text { Chen et al. } \\
(2016)\end{array}$ & $\begin{array}{l}\text { MIT-BIH arrhythmia } \\
\text { dataset }\end{array}$ & $\begin{array}{l}\text { Reduce the } \\
\text { computing time by a } \\
\text { simple method }\end{array}$ & $\begin{array}{l}\text { Wavelet Artificial } \\
\text { Neural Network } \\
\text { (W-ANN) }\end{array}$ & $\begin{array}{l}\text { The average computing } \\
\text { time can be reduced by } \\
49 \%\end{array}$ & $\begin{array}{l}\text { Use a mobile real-time } \\
\text { applications to classify ECG }\end{array}$ \\
\hline $\begin{array}{l}\text { Boussee et al. } \\
\text { (2016) }\end{array}$ & $\begin{array}{l}\text { MIT-BIH arrhythmia } \\
\text { dataset }\end{array}$ & $\begin{array}{l}\text { Record, proceed, } \\
\text { and classify ECG } \\
\text { signal }\end{array}$ & $\begin{array}{l}\text { Mel Frequency } \\
\text { Coefficient } \\
\text { Cepstrum } \\
\text { (MFCC)+ANN }\end{array}$ & $\begin{array}{l}\text { Available a robust and } \\
\text { quick classification } \\
\text { system }\end{array}$ & $\begin{array}{l}\text { Build a system to classify } \\
\text { ECG by a combination of } \\
\text { signal processing algorithms }\end{array}$ \\
\hline $\begin{array}{l}\text { Wess et al. } \\
(2017)\end{array}$ & $\begin{array}{l}\text { MIT-BIH arrhythmia } \\
\text { dataset }\end{array}$ & $\begin{array}{l}\text { To present FPGA- } \\
\text { based ECG } \\
\text { arrhythmia detection }\end{array}$ & PCA+ANN & Accuracy $=99.82 \%$ & $\begin{array}{l}\text { Increased the number of } \\
\text { inputs, hidden layer, and } \\
\text { fixed point }\end{array}$ \\
\hline $\begin{array}{l}\text { Pandey et al. } \\
(2018)\end{array}$ & $\mathrm{UCl}$ arrhythmia dataset & $\begin{array}{l}\text { Early and right } \\
\text { identification of } \\
\text { cardiac disease }\end{array}$ & $\begin{array}{l}\text { RNN, RBF and } \\
\text { BPA }\end{array}$ & $\begin{array}{l}\text { Accuracy } \mathrm{RNN}=83.05 \% \\
\mathrm{RBF}=75.25 \% \\
\mathrm{BPA}=74.35 \%\end{array}$ & $\begin{array}{l}\text { Accuracy of RNN is better } \\
\text { than two ANN models }\end{array}$ \\
\hline $\begin{array}{l}\text { Debnath et al. } \\
\text { (2019) }\end{array}$ & $\begin{array}{l}\text { MIT-BIH arrhythmia } \\
\text { dataset }\end{array}$ & $\begin{array}{l}\text { Analyze and Predict } \\
\text { heart abnormality }\end{array}$ & ANN & $\begin{array}{l}\text { Accuracy } \\
\text { Normal }=97.46 \% \\
\text { Bradycardia }=87.20 \% \\
\text { Tachycardia }=99.97 \% \\
\text { Block }=66.72 \%\end{array}$ & Input noisy ECG signals \\
\hline $\begin{array}{l}\text { Abdalla et al. } \\
(2019)\end{array}$ & $\begin{array}{l}\text { MIT-BIH arrhythmia } \\
\text { dataset }\end{array}$ & $\begin{array}{l}\text { Distinguish between } \\
\text { different types of } \\
\text { ECG arrhythmia }\end{array}$ & CEEMDAN+ANN & Accuracy $=99.9 \%$ & $\begin{array}{l}\text { The performance of the } \\
\text { CEEMDAN and ANN is better } \\
\text { than all existing methods }\end{array}$ \\
\hline
\end{tabular}


TABLE 2: Heartbeat methods classification based on CNN

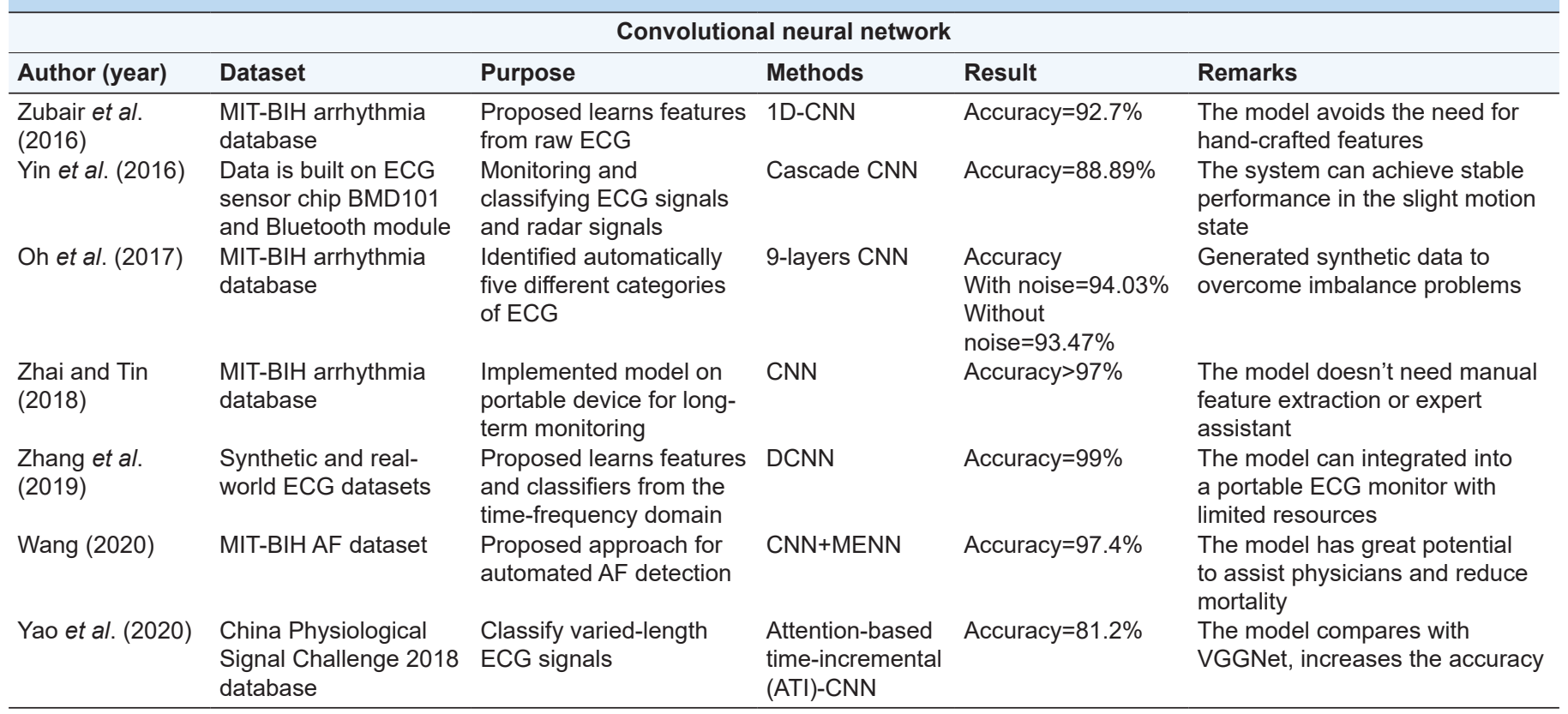

TABLE 3: Heartbeat methods classification based on DWT

\begin{tabular}{|c|c|c|c|c|c|}
\hline \multicolumn{6}{|c|}{ Discrete wavelet transforms } \\
\hline Author (year) & Dataset & Purpose & Methods & Result & Remarks \\
\hline $\begin{array}{l}\text { Desai et al. } \\
(2015)\end{array}$ & $\begin{array}{l}\text { MIT-BIH arrhythmia } \\
\text { dataset }\end{array}$ & $\begin{array}{l}\text { Detected five classes of } \\
\text { ECG arrhythmia }\end{array}$ & $\mathrm{DWT}+\mathrm{ICA}+\mathrm{SVM}$ & Accuracy $=98.49 \%$ & $\begin{array}{l}\text { efficient system in } \\
\text { healthcare diagnosis }\end{array}$ \\
\hline $\begin{array}{l}\text { Saraswat et al. } \\
\text { (2016) }\end{array}$ & $\begin{array}{l}\text { MIT-BIH arrhythmia } \\
\text { dataset }\end{array}$ & $\begin{array}{l}\text { Presented a clear difference } \\
\text { between normal and } \\
\text { abnormal ECG }\end{array}$ & DWT & $\begin{array}{l}\text { Provide min and max } \\
\text { values of normal and } \\
\text { abnormal ECG. }\end{array}$ & $\begin{array}{l}\text { detecting changes in } \\
\text { signals leading to smooth } \\
\text { the feature }\end{array}$ \\
\hline $\begin{array}{l}\text { Alickovic and } \\
\text { Subasi (2016) }\end{array}$ & $\begin{array}{l}\text { MIT-BIH arrhythmia } \\
\text { and St. -Petersburg } \\
\text { Institute of } \\
\text { Cardiological Technics } \\
\text { Arrhythmia Database }\end{array}$ & $\begin{array}{l}\text { Automated system for the } \\
\text { classification of ECG }\end{array}$ & $\mathrm{DWT}+\mathrm{C} 4.5+\mathrm{CART}$ & $\begin{array}{l}\text { Accuracy } \\
\text { C4.5=99.95\% } \\
\text { CART }=99-80 \%\end{array}$ & $\begin{array}{l}\text { Efficient system for } \\
\text { cardiac arrhythmia } \\
\text { detection }\end{array}$ \\
\hline $\begin{array}{l}\text { Pan et } \\
\text { al.(2017) }\end{array}$ & $\begin{array}{l}\text { MIT-BIH arrhythmia } \\
\text { dataset }\end{array}$ & $\begin{array}{l}\text { Developed system } \\
\text { for clinical arrhythmia } \\
\text { classification }\end{array}$ & DWT+random forest & Accuracy=99.77\% & $\begin{array}{l}\text { The system improves } \\
\text { classification accuracy } \\
\text { and speed }\end{array}$ \\
\hline $\begin{array}{l}\text { Sahoo et al. } \\
(2017)\end{array}$ & $\begin{array}{l}\text { MIT-BIH arrhythmia } \\
\text { dataset }\end{array}$ & $\begin{array}{l}\text { Improved algorithm to } \\
\text { detect QRS complex } \\
\text { features to classify four } \\
\text { types of ECG }\end{array}$ & $\begin{array}{l}\text { Multiresolution WT } \\
+\mathrm{NN}+\mathrm{SVM}\end{array}$ & $\begin{array}{l}\text { Accuracy } \\
\text { NN=96.67\% } \\
\text { SVM }=98.39 \%\end{array}$ & $\begin{array}{l}\text { Extracted features are } \\
\text { acceptable for classifying } \\
\text { ECG by SVM }\end{array}$ \\
\hline Ceylan (2018) & $\begin{array}{l}\text { MIT-BIH arrhythmia } \\
\text { dataset }\end{array}$ & $\begin{array}{l}\text { system for signal } \\
\text { compression, noise } \\
\text { elimination, and } \\
\text { classification }\end{array}$ & $\begin{array}{l}\text { DWT+AdaBoost+ } \\
\text { SVM+LDA+DL }\end{array}$ & Accuracy > 99\% & $\begin{array}{l}\text { The best classification } \\
\text { accuracy was obtained by } \\
\text { (DL-AdaBoost - SVM) }\end{array}$ \\
\hline $\begin{array}{l}\text { Tea and } \\
\text { Vladan (2018) }\end{array}$ & $\begin{array}{l}\text { MIT-BIH benchmark } \\
\text { arrhythmia dataset }\end{array}$ & Monitor ECG in real -time & $\begin{array}{l}\mathrm{FFT}+\mathrm{DCT}+\mathrm{DWT} \\
\text { +random forests }\end{array}$ & Accuracy=97.33\% & $\begin{array}{l}\text { DWT provides the best } \\
\text { performance in comparison } \\
\text { with FFT and DCT }\end{array}$ \\
\hline $\begin{array}{l}\text { Zhang et al. } \\
(2019)\end{array}$ & $\begin{array}{l}\text { MIT-BIH arrhythmia } \\
\text { dataset }\end{array}$ & $\begin{array}{l}\text { Diagnosis of lowecost } \\
\text { wearable ECG device }\end{array}$ & DWT+RF+KNN+J48 & Accuracy=98.08\% & $\begin{array}{l}\text { Reduce the computational } \\
\text { cost and improves the } \\
\text { classification efficiency. }\end{array}$ \\
\hline $\begin{array}{l}\text { Kora et al. } \\
(2019)\end{array}$ & $\begin{array}{l}\text { MIT-BIH arrhythmia } \\
\text { dataset }\end{array}$ & $\begin{array}{l}\text { Detect Atrial Fibrillation in } \\
\text { the ECG signal }\end{array}$ & DWT+KNN+SVM & $\begin{array}{l}\text { Accuracy } \\
\text { DWT+SVM=94.07\% } \\
\text { DWT+KNN=99.5\% }\end{array}$ & $\begin{array}{l}\text { DWT represent the } \\
\text { essential characteristics } \\
\text { of the ECG }\end{array}$ \\
\hline
\end{tabular}


TABLE 4: Heartbeat methods classification based on SVM

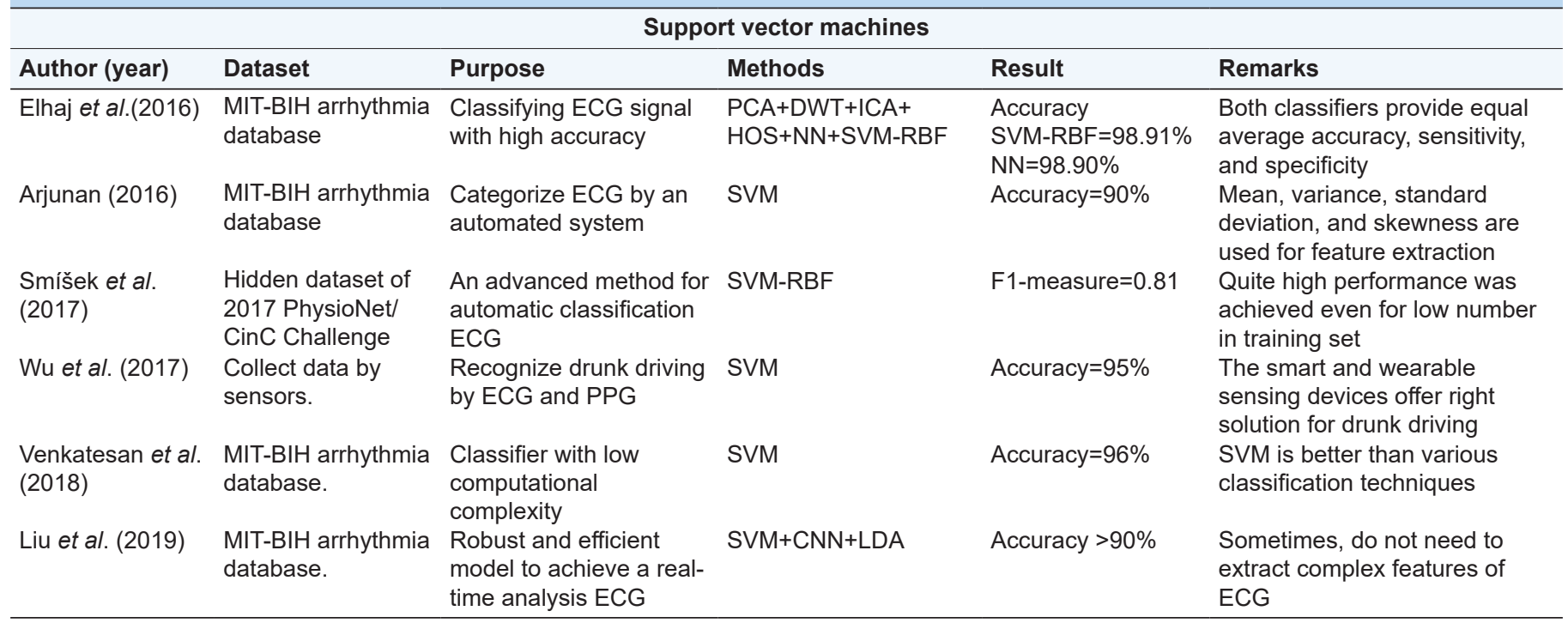

TABLE 5: Heartbeat methods classification based on KNN

\begin{tabular}{|c|c|c|c|c|c|}
\hline \multicolumn{6}{|c|}{ K- nearest neighbors } \\
\hline Author (year) & Dataset & Purpose & Methods & Result & Remarks \\
\hline $\begin{array}{l}\text { Faziludeen and } \\
\text { Sankaran(2016) }\end{array}$ & $\begin{array}{l}\text { MIT-BIH arrhythmia } \\
\text { database }\end{array}$ & $\begin{array}{l}\text { Classify ECG beat } \\
\text { into two classes }\end{array}$ & $\mathrm{KNN}+\mathrm{EKNN}$ & Lower error rates & $\begin{array}{l}\text { Increase in training size is } \\
\text { shown to lower the error rates }\end{array}$ \\
\hline $\begin{array}{l}\text { Khatibi and } \\
\text { Rabinezhadsadatmahaleh } \\
\text { (2019) }\end{array}$ & $\begin{array}{l}\text { MIT-BIH arrhythmia } \\
\text { database }\end{array}$ & $\begin{array}{l}\text { Classify ECG for } \\
\text { arrhythmia detection }\end{array}$ & $\begin{array}{l}\text { CNN+DT+S } \\
\text { VM+RF+K-NNs }\end{array}$ & Accuracy $=99.77 \%$ & $\begin{array}{l}\text { The method is low } \\
\text { computational time }\end{array}$ \\
\hline
\end{tabular}

classification and which kind of ECG datasets were used. Some important points in the ECG classification are observed and highlighted in the below:

According to the previous works based on the ANN algorithm for heartbeat classification, ANN is trained using the polyspectrum patterns and features extracted from the higher-order spectral analysis of normal and abnormal ECG signal. ANN is used as a classifier to help knowledge management and decision-making system to improve classification ACC. The result shows that ANN with PCA obtains lowest error rate to classify the ECG signal. The performance of the CEEMDAN and ANN is better than all higher than all existing and previous algorithms (Table 1). (The main point are extracted from ANN [Reviewer 1 and 2 and 3]).

$\mathrm{CNN}$ is straight forward to apply as the $\mathrm{CNN}$ is a features less techniques. Hence, the researcher does not concern about the feature that means any handcraft feature does not require in the $\mathrm{CNN}$ model. $1 \mathrm{D}$ and $2 \mathrm{D}$ of $\mathrm{CNN}$ have been adopted, According to the observed result, $1 \mathrm{D}$ CNN outperformed of the 2D CNN. Moreover, the 1D $\mathrm{CNN}$ is less complex compare to the $2 \mathrm{D} \mathrm{CNN}$ in term of computational steps. CNN also can be integrated with MENN to improve the classification ACC (Table 2). (Roles $\mathrm{CNN}$ in ECG classification [Reviewer 1 and 2 and 3]).

DWT is applied on each heartbeat to obtain the morphological features. It provides better time and frequency resolution of ECG signal. DWT shows the powerful tool for ECG classification and it is straight forward tool to implantation. Moreover, DWT is an assisting the clinicians for making an accurate diagnosis of CVDs. Based on the summarization of some works on DWT, the integration DWT model with random forest can achieve $99.77 \%$ ACC (Table 3) (The main notes about DWT [Reviewer 1 and 2 and 3]).

SVM (SVM) is widely used for pattern recognition. SVM model with a weighted kernel function method significantly 
recognizes the $\mathrm{Q}$ wave, $\mathrm{R}$ wave, and $\mathrm{S}$ wave in the input $\mathrm{ECG}$ signal to categorize the heartbeat. SVM is also the powerful tool to ECG classification; however, the performance CNN has outperformed of the SVM. Moreover, the time consumption of implementing SVM is higher than KNN model and smaller than the CNN model. SVM-RBF classifier classifies $95 \%$ of the given ECG signal correctly with simple statistical features Table4. (The contributions of SVM [Reviewer 1 and 2 and 3]).

The lowest computational rate for diagnosing arrhythmia can be achieved by applying KNN as the KNN algorithm does not require the training stage. The role of the handcraft features is a vital subject to the KNN model as long as the dimensional of the obtained features is low because the KNN model works based on the distance. Time domain and frequency domain features are applied to $\mathrm{KNN}$ classifier for ECG classification which is simpler than other machinelearning approaches (Table 5). (The main roles of $\mathrm{kNN}$ in ECG classification [Reviewer 1 and 2 and 3]).

\section{CONCLUSION}

Classification of ECG signals is acting an important role in recognizing normal and abnormal heartbeat. Increasing the ACC of ECG classification is a challenging problem. It has been interested in more than a decade; for this reason, many approaches have been developed. In this paper, most recent approaches are reviewed in terms of some aspects such as method, dataset, contribution, and success rate. The table (CNN) summarizes variance approaches in ECG signal analysis. We suggest using a hybrid model based on CNN with long- and short-term memory (LSTM). The CNN part can extract the features from the raw signal which can be a temporal features based on how many convolution layers we will used, and LSTM can learn the pattern in the temporal feature as the LSTM is more suitable to time series features. Then, the model can predict unknown ECG signals. We will tune filters in the CNN model and layers in the LSTM model to increase the classification rate. (Explain how use CNN+LSTM [Reviewer 3]).

\section{REFERENCES}

[1] A. Alberdi, A. Aztiria and A. Basarab. "Towards an automatic early stress recognition system for office environments based on multimodal measurements: A review". Journal of Biomedical Informatics, vol. 59, pp. 49-75, 2016.

[2] M. S. Al-Ani. "Electrocardiogram waveform classification based on P-QRS-T wave recognition". UHD Journal of Science and
Technology, vol. 2, no. 2, pp. 7-14, 2018.

[3] M. Al-Ani. "A rule-based expert system for automated ecg diagnosis". International Journal of Advances in Engineering and Technology, vol. 6, no. 4, 1480-1492, 2014.

[4] M. S. Al-Ani and A. A. Rawi. "ECG Beat diagnosis approach for ECG printout based on expert system". International Journal of Emerging Technology and Advanced Engineering, vol. 3, no. 4, pp. 797-807, 2013.

[5] S. H. Jambukia, V. K. Dabhi and H. B. Prajapati. "Classification of ECG Signals Using Machine Learning Techniques: A Survey". In: Conference Proceeding 2015 International Conference on Advances in Computer Engineering and Applications, pp. 714-721, 2015.

[6] J. Li, Y. Si, T. Xu and S. Jiang. "Deep convolutional neural network based ecg classification system using information fusion and onehot encoding techniques". Mathematical Problems Engineering, vol. 2018, p. 7354081, 2018.

[7] D. Sung, J. Kim, M. Koh and K. Park. "ECG Authentication in postexercise situation ECG authentication in post-exercise situation". Conference Proceeding IEEE Engineering Medical Biology Socirty, vol. 1, pp. 446-449, 2017.

[8] M. Lakshmi, D. Prasad and D. Prakash. "Survey on EEG signal processing methods". International Journal of Advanced Research in Computer Science, vol. 4, no. 1, pp. 84-91, 2014.

[9] R. Chaturvedi and Y. Yadav. "A survey on compression techniques". International Journal of Advanced Research in Computer and Communication Engineering, vol. 2, no. 9, pp. 3511-3513, 2013.

[10] H. Y. Lin, S. Y. Liang, Y. L. Ho, Y. H. Lin and H. P. Ma. "Discretewavelet-transform-based noise removal and feature extraction for ECG signals". IRBM, vol. 35, no. 6, pp. 351-361, 2014.

[11] M. Hammad, S. Zhang and K. Wang. "A novel two-dimensional ECG feature extraction and classification algorithm based on convolution neural network for human authentication". Future Generation Computer Systems, vol. 101, pp. 180-196, 2019.

[12] J. Juang. "Proceedings of the $3^{\text {rd }}$ International Conference on Intelligent Technologies and Engineering Systems (ICITES2014)". Vol. 345. In: Lecture Notes in Electrical Engineering, pp. 545-555, 2016.

[13] A. Giorgio, M. Rizzi and C. Guaragnella. "Efficient detection of ventricular late potentials on ECG signals based on wavelet denoising and SVM classification". Information, vol. 10, no. 11, p. 328, 2019.

[14] F. A. R. Sánchez and J. A. G. Cervera. "ECG classification using artificial neural networks". Journal of Physics: Conference Series, vol. 1221, no. 1, pp. 1-6, 2019.

[15] S. V. Deshmukh and O. Dehzangi. "ECG-Based Driver Distraction Identification Using Wavelet Packet Transform and Discriminative Kernel-Based Features". 2017 IEEE International Conference on Smart Computing, 2017.

[16] S. K. Berkaya, A. K. Uysal, E. S. Gunal, S. Ergin, S. Gunal and M. B. Gulmezoglu. "A survey on ECG analysis". Biomedical Signal Processing and Control, vol. 43, pp. 216-235, 2018.

[17] N. A. Polytechnic. "Automated identification of shockable and non-shockable life-threatening ventricular arrhythmias using convolutional neural network". Future Generation Computer Systems the International Journal of Escience, vol. 79, p. 952, 2017.

[18] F. A. Elhaj, N. Salim, T. Ahmed, A. R. Harris and T. T. Swee. "Hybrid Classification of Bayesian and Extreme Learning Machine for Heartbeat Classification of Arrhythmia Detection". In: $6^{\text {th }}$ ICT 
International Student Project Conference, pp. 1-4, 2017.

[19] P. Li, K. L. Chan, S. Fu and S. M. Krishnan. "An abnormal ECG beat detection approach for long-term monitoring of heart patients based on hybrid kernel machine ensemble". Lecture Notes in Computer Science, Vol. 354. 1Springer, Berlin, pp. 346-355, 2005.

[20] S. Shadmand and B. Mashoufi. "A new personalized ECG signal classification algorithm using block-based neural network and particle swarm optimization". Biomedical Signal Processing and Control, vol. 25, pp. 12-23, 2016.

[21] J. Mateo, A. M. Torres, A. Aparicio and J. L. Santos. "An efficient method for ECG beat classification and correction of ectopic beats". Computers and Electrical Engineering, vol. 53, pp. 219229, 2016.

[22] C. Kamalakannan, L. P. Suresh, S. S. Dash and B. K. Panigrahi. "Power Electronics and Renewable Energy Systems: Proceedings of ICPERES 2014. Vol. 326. Lecture Notes in Electrical Engineering, pp. 1537-1544, 2014.

[23] C. K. and B. S. "A survey on various machine learning approaches for ECG analysis". International Journal of Computer Applications, vol. 163, no. 9, pp. 25-33, 2017.

[24] S. Z. Islam, S. Z. Islam, R. Jidin and M. A. M. Ali. "Performance Study of Adaptive Filtering Algorithms for Noise Cancellation of ECG Signal". Vol. 4. In: ICICS 2009 Conference Proceeding $7^{\text {th }}$ International Conference Information, Communication Signal Process, 2009.

[25] M. A. Rahman, M. M. Milu, A. Anjum, A. B. Siddik, M. H. Sifat, M. R. Chowdhury, F. Khanam, M. Ahmad. "A statistical designing approach to MATLAB based functions for the ECG signal preprocessing". The Iran Journal of Computer Science, vol. 2, no. 3, pp. 167-178, 2019.

[26] M. T. Almalchy, V. Ciobanu and N. Popescu. "Noise removal from ECG signal based on filtering techniques". Proceeding $201922^{\text {nd }}$ International Conference Control Systems Computer Science, pp. 176-181, 2019.

[27] G. H. Choi, E. S. Bak and S. B. Pan. "User identification system using $2 \mathrm{D}$ resized spectrogram features of ECG". IEEE Access, vol. 7, pp. 34862-34873, 2019.

[28] A. Lay-Ekuakille, M. A. Ugwiri, C. Liguori and P. K. Mvemba. "Enhanced methods for extracting characteristic features from ECG". IEEE International Symposium on Medical Measurements and Applications, pp. 1-5, 2019.

[29] J. Oster, J. Behar, O. Sayadi, S. Nemati, A. E. W. Johnson and G. D. Clifford. "Semisupervised ECG ventricular beat classification with novelty detection based on switching kalman filters". IEEE Transactions on Biomedical Engineering, vol. 62, no. 9, pp. 21252134, 2015.

[30] S. Karpagachelvi. "ECG feature extraction techniques a survey approach". International Journal of Computer Science and Information Security, vol. 8, no. 1, pp. 76-80, 2010.

[31] S. Nasehi and H. Pourghassem. "Seizure detection algorithms based on analysis of EEG and ECG signals: A survey". Neurophysiology, vol. 44, no. 2, pp. 174-186, 2012.

[32] S. M. J. Jalali, M. Karimi, A. Khosravi and S. Nahavandi. "An efficient neuroevolution approach for heart disease detection". Conference Proceeding IEEE International Conference System Man Cybernetics, pp. 3771-3776, 2019.

[33] D. Carrera, B. Rossi, P. Fragneto and G. Boracchi. "Online anomaly detection for long-term ECG monitoring using wearable devices". Pattern Recognition, vol. 88, pp. 482-492, 2019.

[34] E. K. Wang, X. Zhang and L. Pan. "Automatic classification of CAD
ECG signals with SDAE and bidirectional long short-term network". IEEE Access, vol. 7, pp. 182873-182880, 2019.

[35] N. Omer, Y. Granot, M. Kähönen, R. Lehtinen, T. Nieminen, K. Nikus. "Blinded analysis of an exercise ECG database using high frequency QRS analysis". Vol. 44. In: 2017 Computing in Cardiology, pp. 1-4, 2017.

[36] I. Karagoz. "Cmbebih 2019". Vol. 73. In: IFMBE Proceeding C, pp. 159-163, 2019.

[37] S. Lata and R. Kumar. "Disease classification using ECG signals based on R-peak analysis with ABC and ANN". The International Journal of Electronics, Communications, and Measurement Engineering, vol. 8, no. 2, pp. 67-86, 2019.

[38] A. Delrieu, M. Hoël, C. T. Phua and G. Lissorgues. "Multi physiological signs model to enhance accuracy of ECG peaks detection". IFMBE Proceeding, vol. 61, pp. 58-61, 2017.

[39] K. C. J. Chen, Y. S. Ni and J. Y. Wang. "Electrocardiogram Diagnosis Using Wavelet-Based Artificial Neural Network". In: 2016 IEEE $5^{\text {th }}$ Globel Conference Consumer Electronics GCCE 2016, pp. 5-6, 2016.

[40] M. Boussaa, I. Atouf, M. Atibi and A. Bennis. "ECG Signals Classification Using MFCC Coefficients and ANN Classifier". Proceeding 2016 International Conference Electronics Information Technology, pp. 480-484, 2016.

[41] S. Savalia, E. Acosta and V. Emamian. "Classification of cardiovascular disease using feature extraction and artificial neural networks". Journal of Biosciences and Medicines, vol. 5, no. 11, pp. 64-79, 2017.

[42] M. Wess, P. D. S. Manoj and A. Jantsch. "Neural Network Based ECG Anomaly Detection on FPGA and Trade-off Analysis. In: Proceedings IEEE International Symposium on Circuits and Systems, 2017.

[43] S. Pandey and R. R. Janghel. "Classification of ECG arrhythmia using recurrent neural networks ECG arrhythmia classification using artificial neural networks". Procedia Computer Science, vol. 8, pp. 1290-1297, 2018.

[44] G. Sannino and G. De Pietro. "A deep learning approach for ECGbased heartbeat classification for arrhythmia detection". Future Generation Computer Systems, vol. 86, pp. 446-455, 2018.

[45] T. Debnath, M. Hasan and T. Biswas. "Analysis of ECG Signal and Classification of Heart Abnormalities Using Artificial Neural Network". In: Proceeding $9^{\text {th }}$ International Conference Electrical and Computer Engineering, pp. 353-356, 2017.

[46] F. Y. O. Abdalla, L. Wu, H. Ullah, G. Ren, A. Noor and Y. Zhao. "ECG arrhythmia classification using artificial intelligence and nonlinear and nonstationary decomposition". Signal, Image Video Process, vol. 13, no. 7, pp. 1283-1291, 2019.

[47] Z. K. Abdul. "Kurdish speaker identification based on one dimensional convolu- tional neural network". Computational Methods for Differential Equations, vol. 7, no. 4, pp. 566-572, 2019.

[48] D. Li, J. Zhang, Q. Zhang and X. Wei. "Classification of ECG signals based on 1D convolution neural network". In: 2017 IEEE $19^{\text {th }}$ International Conference on e-Health Networking, Applications and Services, Healthcom, pp. 1-6, 2017.

[49] M. Zubair, J. Kim and C. Yoon. "An automated ECG beat classification system using convolutional neural networks". In: $20166^{\text {th }}$ International Conference on IT Convergence and Security, 2016.

[50] W. Yin, X. Yang, L. Zhang and E. Oki. "ECG monitoring system integrated with IR-UWB radar based on CNN". IEEE Access, vol. 
4, pp. 6344-6351, 2016.

[51] S. L. Oh, N. A. Polytechnic, N. A. Polytechnic, Y. Hagiwara and J. H. Tan. "A deep convolutional neural network model to classify heartbeats". Computers in Biology and Medicine, vol. 89, pp. 389396, 2017.

[52] X. Zhai and C. Tin. "Automated ECG classification using dual heartbeat coupling based on convolutional neural network". IEEE Access, vol. 6, pp. 27465-27472, 2018.

[53] J. Zhang, J. Tian, Y. Cao, Y. Yang and X. Xu. "Deep time frequency representation and progressive decision fusion for ECG classification". Knowledge-Based Systems, vol. 190, p. 105402, 2020.

[54] J. Wang. "A deep learning approach for atrial fibrillation signals classification based on convolutional and modified Elman neural network". Future Generation Computer Systems, vol. 102, pp. 670679, 2020.

[55] Q. Yao, R. Wang, X. Fan, J. Liu and Y. Li. "Multi-class arrhythmia detection from 12-lead varied-length ECG using attention-based time-incremental convolutional neural network". Information Fusion, vol. 53, no. 1, pp. 174-182, 2020.

[56] H. Limaye and V. V. Deshmukh. "ECG noise sources and various noise removal techniques: A survey". International Journal of Application or Innovation in Engineering and Management, vol. 5, no. 2, pp. 86-92, 2016.

[57] S. L. Joshi. "A Survey on ECG Signal Denoising Techniques 2013 International Conference on Communication Systems and Network Technologies A Survey on ECG Signal DenoisingTechniques", 2013.

[58] H. El-Saadawy, M. Tantawi, H. A. Shedeed and M. F. Tolba. "Electrocardiogram (ECG) classification based on dynamic beats segmentation". The ACM International Conference Proceeding Series, pp. 75-80, 2016.

[59] T. R. Naveen, K. V. Reddy, A. Ranjan and S. Baskaran. "Detection of abnormal ECG signal using DWT feature extraction and CNN". International Research Journal of Engineering and Technology, vol. 6, no. 3, pp. 5175-5180, 2019.

[60] U. Desai, R. J. Martis, C. G. Nayak, K. Sarika and G. Seshikala. "Machine Intelligent Diagnosis of ECG for Arrhythmia Classification Using DWT, ICA and SVM Techniques. 12 ${ }^{\text {th }}$ IEEE International Conference Electronic Energy, Environmental Research Communications, pp. 2-5, 2016.

[61] S. Saraswat, G. Srivastava and S. Shukla. "Decomposition of ECG Signals using Discrete Wavelet Transform for Wolff Parkinson White Syndrome Patients". In: Proceedings 2016 International Conference on Micro-Electronics and Telecommunication Engineering, pp. 361-365, 2016.

[62] E. Alickovic and A. Subasi. "Medical decision support system for diagnosis of heart arrhythmia using DWT and random forests classifier". The Journal of Medical Systems, vol. 40, no. 4, pp. 1-12, 2016.

[63] G. Pan, Z. Xin, S. Shi and D. Jin. "Arrhythmia classification based on wavelet transformation and random forests". Multimedia Tools and Applications Journal, vol. 77, no. 17, pp. 21905-21922, 2018.

[64] S. Sahoo, B. Kanungo, S. Behera and S. Sabut. "Multiresolution wavelet transform based feature extraction and ECG classification to detect cardiac abnormalities Multiresolution wavelet transform based feature extraction and ECG classification to detect cardiac abnormalities". Measurement, vol. 17, no. 1, pp. 55-66, 2017.

[65] M. Barstuğan and R. Ceylan. "The effect of dictionary learning on weight update of AdaBoost and ECG classification". Journal of
King Saud University, vol. 30, pp.1-9, 2018.

[66] T. Marasović and V. Papić. "A comparative study of FFT, DCT, and DWT for efficient arrhytmia classification in RP-RF framework". International Journal of E-Health and Medical Communications, vol. 9, no. 1, pp. 35-49, 2018.

[67] Y. Zhang, Y. Zhang, B. Lo and W. Xu. "Wearable ECG signal processing for automated cardiac arrhythmia classification using CFASE-based feature selection". Expert System, vol. 37, no. 1, pp. 1-13, 2020.

[68] P. Kora, C. U. Kumari, K. Swaraja and K. Meenakshi. "Atrial Fibrillation detection using Discrete Wavelet Transform. In: Proceedings of $20193^{\text {rd }}$ IEEE International Conference on Electrical, Computer and Communication Technologies, pp. 1-3, 2019.

[69] S. Raj and K. C. Ray. "ECG signal analysis using DCT-Based DOST and PSO Optimized SVM". IEEE Transactions on Automatic Control, vol. 66, no. 3, pp. 470-478, 2017.

[70] R. Banerjee, A. Ghose and S. Khandelwal. "A Novel Recurrent Neural Network Architecture for Classification of Atrial Fibrillation Using Single-lead ECG. In: European Signal Processing Conference, pp. 1-5, 2019.

[71] H. Khorrami and M. Moavenian. "A comparative study of DWT, CWT and DCT transformations in ECG arrhythmias classification". Expert Systems With Applications, vol. 37, no. 8, pp. 5751-5757, 2010.

[72] V. Mygdalis, A. Tefas and I. Pitas. "Exploiting multiplex data relationships in support vector machines". Pattern Recognition, vol. 85, pp. 70-77, 2019.

[73] F. A. Elhaj, N. Salim, A. R. Harris, T. T. Swee and T. Ahmed. "Arrhythmia recognition and classification using combined linear and nonlinear features of ECG signals". Computer Methods and Programs in Biomedicine, vol. 127, pp. 52-63, 2016.

[74] V. R. Arjunan. "ECG signal classification based on statistical features with SVM classification". International Journal of Advances in Signal and Image Sciences, vol. 2, no. 1, p. 5, 2016.

[75] R. Smíšek, J. Hejč, M. Ronzhina, A. Němcová, L. Maršánová, J. Chmelík, K. Jana. SVM Based ECG classification using rhythm and morphology features, cluster analysis and multilevel noise estimation". Computing in Cardiolology, vol. 44, pp. 1-4, 2017.

[76] W. F. Wang, C. Y. Yang and Y. F. Wu. "SVM-based classification method to identify alcohol consumption using ECG and PPG monitoring". Personal and Ubiquitous Computing, vol. 22, no. 2, pp. 275-287, 2018.

[77] C. Venkatesan, P. Karthigaikumar, A. Paul, S. Satheeskumaran and R. Kumar. "ECG signal preprocessing and SVM classifierbased abnormality detection in remote healthcare applications". IEEE Access, vol. 6, pp. 9767-9773, 2018.

[78] J. Liu, S. Song, G. Sun and Y. Fu. "Classification of ECG arrhythmia Using CNN, SVM and LDA". Vol. 11633. In: International Conference on Artificial Intelligence and Security, pp. 191-201, 2019.

[79] J. Zhai and A. Barreto. "Stress Detection in Computer Users Based on Digital Signal Processing of Noninvasive Physiological Variables". In: Proceedings of the 28 th IEEE EMBS Annual International Conference, pp. 1355-1358, 2007.

[80] V. Gupta and M. Mittal. "KNN and PCA classifier with Autoregressive modelling during different ECG signal interpretation". Procedia Computer Science, vol. 125, pp. 18-24, 2018.

[81] N. Flores, R. L. Avitia, M. A. Reyna and C. García. "Readily available ECG databases". Journal of Electrocardiology, vol. 51, 
no. 6, pp. 1095-1097, 2018.

[82] R. P. Narwaria, S. Verma and P. K. Singhal. "Removal of baseline wander and power line interference from ECG signal a survey approach". International Journal of Information and Electronics Engineering, vol. 3, no. 1, pp. 107-111, 2011.

[83] N. K. Dewangan and S. P. Shukla. "A survey on ECG signal feature extraction and analysis techniques". International Journal of Innovative Research in Electrical, Electronics, Instrumentation and Control Engineering, vol. 3, no. 6, pp. 12-19, 2015.

[84] I. Saini. "Analysis ECG data compression techniques a survey approach". The International Journal of Emerging Technology and Advanced Engineering, vol. 3, no. 2, pp. 544-548, 2013.

[85] M. M. Baig, H. Gholamhosseini and M. J. Connolly. "A comprehensive survey of wearable and wireless ECG monitoring systems for older adults". Medical and Biological Engineering and Computing, vol. 51, no. 5, pp. 485-495, 2013.

[86] S. Faziludeen and P. Sankaran. "ECG beat classification using evidential K-nearest neighbours". Procedia Computer Science, vol. 89, pp. 499-505, 2016

[87] F. Bouaziz, D. Boutana and H. Oulhadj. "Diagnostic of ECG Arrhythmia Using Wavelet Analysis and K-Nearest Neighbor Algorithm". In: Proceedings of the 2018 International Conference on Applied Smart Systems, pp. 1-6, 2019.

[88] T. Khatibi and N. Rabinezhadsadatmahaleh. "Proposing feature engineering method based on deep learning and K-NNs for ECG beat classification and arrhythmia detection". Physical and Engineering Sciences in Medicine, vol. 43, pp. 1-20, 2019. 